This is an Accepted Manuscript of an article published by Taylor \& Francis in International Journal of Construction Education and Research on 08/05/17, available online: https://www.tandfonline.com/doi/ full/10.1080/15578771.2017.1316799 


\title{
UNDERSTANDING THE UNDERREPRESENTATION OF QUALITATIVE RESEARCH APPROACHES TO BUILT ENVIRONMENT RESEARCH IN NIGERIA
}

\author{
Umeokafor Nnedinma \\ Department of Built Environment, Faculty of Architecture, Computing \& Humanities, \\ University of Greenwich, London, UK \\ Windapo, Abimbola Olukemi \\ Department of Construction Economics and Management, University of Cape Town, \\ Cape Town, South Africa
}

\begin{abstract}
There is evidence that scholars in some countries, which includes Nigeria, mostly employ the quantitative approach to research, and in some cases it is used inappropriately. This leaves research questions that should be tackled from a qualitative standpoint, unaddressed or wrongly undertaken. The aim of this study is to understand why the qualitative approach (QA) has failed to gain similar recognition in Nigeria and other countries, and by extension, the disposition of researchers towards its use. The impact of the disposition of researchers towards QA was also explored. Researchers in the various built environment (BE) disciplines in Nigeria were interviewed. The results revealed that there is a preference for quantitative research, while QAs are used if convenient. Furthermore, the study revealed that the educational background of academics and their poor understanding of the qualitative paradigm, explain their disposition towards the adoption of the QA in research. This finding suggests that academic followers instead of leaders are being produced, and that research is done for ad hominem promotion. In advancing the understanding of QA in BE research in Nigeria, this study also draws the attention of stakeholders in the academia to the implications of a preference by researchers for quantitative research.
\end{abstract}

Keywords: built environment, interpretivism, Nigeria, qualitative research, research methodology.

\section{INTRODUCTION}

Although $\mathrm{BE}$ research has grown and developed over the years after the methodological debate in the mid-1990s (Dainty 2007A), there is still concern about the lack of uniformity or imbalance in the adopted methodologies - quantitative and qualitative (Carter \& Fortune 2004; Dainty, Bagilhole \& Neale 1997; Danity 2007A B; Ejohwomu \& Oshodi 2014; Laryea \& Leiringer 2012; Loosemore, Hall \& Dainty 1996; Panas \& Pantouvakis 2010). Quantitative research is of positivist, emperialist and rationalist philosophical positions, while qualitative research is mainly of an interpretivist or constructivist philosophical positions, but not absolutely. Strauss and Corbin (1997:17) state that qualitative research is "...any kind of research that produces findings not arrived at by means of statistical procedures or other means of qualification." Thus, reality is a variable social construct and is dependent on the researcher. This involved research methods such as observations and interviews. In a 
mixed methods approach both the quantitative and the qualitative methods are applied (Creswell 2014); this approach is of a pragmatic philosophical position (Saunders, Lewis, and Thornhill 2009). According to Saunders et al. (2009:109): "Pragmatism argues that the most important determinant of the epistemology, ontology and axiology you adopt is the research question - one may be more appropriate than the other for answering particular questions."

Research methods are conceptualised in this study as data collection, analysis and interpretation methods, while research strategy is used as the "basic philosophical assumptions researchers bring to the study, the procedure of inquiry... called research design" (Creswell 2014:4), for example, archival research, explanatory sequential mixed methods, statistical survey, ethnography. According to Creswell (2014:3-4), research approaches are plans and procedures for the research, ranging from broad assumptions to detailed research methods, for example, quantitative, qualitative, and mixed methods approaches.

Typically, in technology education research in the US, Zuga (1994) found that quantitative methods and analysis are grossly overrepresented. Carter and Fortune (2004) reported that at a conference series held in the UK in 2000 and 2001, and in a research done at Heriot-Watt University UK from 2001-2003, the QA in the BE was underrepresented. This is consistent with other studies such as Danity (2007B), and Panas and Pantouvakis (2010) who also report the underrepresentation of the QA to research in the BE. A similar trend is reported in developing countries. Typically, from a non-built environment perspective, Bubaker, Balakrishnan, and Bernadine (2005), Dzvimbo (1994), and Crossley and Vulliamy (1996) decry the paucity of qualitative research in some developing countries, while from a BE perspective in West Africa, including Nigeria, Ejohwomu and Oshodi (2014), and Laryea and Leiringer (2012) both support this position, evidencing the dominance of the quantitative approach to research, and the underrepresentation of the qualitative approach to research in West Africa, reinforcing the personal observations of the authors of the current paper.

It can be argued that assuming qualitative research to be underrepresented because it does not occupy, as much space as does quantitative research, is a false assumption. However, it should be noted that if this is the case, then there is no need for statistical data that show the level of representation of various matters and no need for descriptive statistics, which forms part of the quantitative approach. If this is the case, it can be argued that the authors of the current paper are not biased toward any particular paradigm, as qualitative research informed the basics for the current research. In other words, their philosophical position is that research questions should be addressed on a fit-for-purpose basis.

Nonetheless, scholars view that there is a strong culture of applying quantitative research in the BE context such as construction management (Carter and Fortune 2004). Hence, according to Hughes (2010), and Laryea and Leiringer (2012), some of the research questions addressed by quantitative methods are still wrongly tackled, using inadequate research methods. The strong culture of quantitative research does not only reflect on what is taught by academics, contributing to developing the research orientation of students and early researchers (Carter and Fortune 2004), it also suggests that the QA to research is unrecognised and maybe unaccepted in some 
countries (Dzvimbo 1994). According to Carter and Fortune (2004:942) and Hughes (2010), students and early researchers are influenced by the culture of the discipline where their supervisors or lecturers are dominant in guiding the research approach and the methods used, and if these supervisors or lecturers have a strong quantitative research culture, Hughes (2010) argues that the disposition of these academics will not only impact on the adoption of other research approaches, but also on how students are taught.

There are however, possible explanations to the underrepresentation of the QA to research and the disposition of academics towards the QA approach. For instance, Dainty (2007A) posits that many BE disciplines are orientated toward engineering which has a quantitative research bias, and this may in part explain the overrepresentation of the quantitative approach to research related to the BE. Also, some challenges posed by qualitative approaches, such as validity and reliability, and measures such as reflectivity, and detailed reporting can improve rigour in the paradigm (see Umeokafor 2015). While Bubaker et al. (2005) highlighted challenges specific to research in developing countries that deters researchers from using the qualitative research approach due to the contextual environment, there is evidence by Kheni (2008), of cultural issues that present opportunities for the use of the qualitative research approach in developing countries.

Notwithstanding, the dominance of quantitative research in and outside the BE, limited research has been undertaken to understand why qualitative research remains underrepresented or why there is a strong culture of quantitative research in the BE. For instance, Dainty (2007A) calls for methodological pluralism to inculcate interpretative research design, so as to achieve a balance in methodological outlook. Earlier, Dainty, Bagilhole and Neale (1997) (in anticipation of the rush by academics in the $\mathrm{BE}$ to employ more interpretivist methodologies due to several calls for greater use of QA) took a proactive step when they discussed analytical strategies for the QA data and how academics can demonstrate reliability and validity in the QA. In addressing this gap in knowledge, this study examines the QA to BE research in Nigeria and the disposition of researchers towards its use, so as to understand why qualitative research remains underrepresented in Nigeria's BE research and by extension to other disciplines, and whether the disposition of academia towards its use, impacts on academics and students. Thus, the implications of the assumed disposition of academics also suggest their understanding of the paradigm. The authors of the current paper view that the current study may contribute to addressing or at least raising awareness of the critical imperativeness of the under-adoption of QA to research. It is acknowledged that although the strong culture for quantitative methods and analysis is not limited to Nigeria and the BE, Nigeria is used as a case study with the BE forming the context of this study.

The sections of the paper following, are the literary perspectives, the presentation of the methodology used in the study, the results, and a discussion. Finally, the paper presents a conclusion section that highlights the deductions and implications of the findings for theory and practice.

\section{LITERATURE DISCUSSION}


The Built Environment research and underrepresentation of qualitative research There is evidence to show an imbalance in the adoption of research approachescin the Built Environment (BE) (see Loosemore et al., 1996; Carter and Fortune, 2004; and Dainty, 2007B). From a non-built environment perspective, particularly in technology education, Zuga (1994) found that of the 220 reports reviewed, only 16 (about 7\%) adopted a qualitative approach to research; about $85 \%$ employed quantitative research methods, with 65\% involving descriptive research. Meanwhile, Hoepfl (1997) contends that people are unfamiliar with qualitative research methodologies and goes on to make a case for adopting the QA in technology education research.

In the built environment, scholars such as Loosemore et al. (1996), Carter and Fortune (2004) and Dainty (2007B) established that quantitative research approaches remain dominant in construction management research. Loosemore et al. (1996) reviewed articles published in the Journal of Construction Management and Economics from 1983 to 1993 and found that papers using qualitative methods and analysis accounted for $8 \%$ of the publications, quantitative methods and analysis papers for $57 \%$, mixed methodology for $13 \%$, and discussion papers for $22 \%$. Furthermore, Carter and Fortune (2004) reviewed papers published for a conference series held in the UK in the years 2000 and 2001, as well as postgraduate research papers from HWU from 2001-2003, and found that qualitative methodology accounted for $18 \%$, quantitative methodology for $42 \%$, mixed methodology for $31 \%$, and discussion papers for $9 \%$ in the HWU research and that on average, qualitative methodology accounted for $13 \%$, quantitative methodology for $29 \%$, mixed methodology for $18 \%$, and discussion papers for $42 \%$ in the conference series.

Dainty (2007A) reviewed papers published in the Journal of Construction Management and Economics, Volume 24 of 2006 and found that the papers are oriented more towards the positivist paradigm. Out of the 107 papers published, 9 used qualitative methods, 76 adopted a quantitative approach, 12 mixed methods, and 10 review/other papers. In a more recent study, Panas and Pantouvakis (2010) reviewed 89 construction productivity papers from 1999 to 2009 in top quality journals and found that 9 (approximately 10\%) were of qualitative approach, 26 (29\%) used mixed methods, and 54 (approximately 61\%) used the quantitative approach. Other scholars such as Hyari, El-Mashaleh and Rababeh (2015) also acknowledge the under-adoption of qualitative methods such as focus group discussion in construction management research.

Furthermore, the strong culture of quantitative research is also reported in the BE context in developing countries as a detriment of knowledge and practice (Bubaker et al. 2005; Crossley \& Vulliamy 1996; Dzvimbo 1994; Hughes 2010; Laryea \& Leiringer 2012). Authors such as Bubaker et al. (2005), Crossley and Vulliamy (1996), and Dzvimbo (1994) note and demonstrate the dominance of positivist strategies in developing countries. Covering developing countries such as Libya and Malaysia, Bubaker et al. (2005) showed how the QA to research remains underadopted, while statistical survey research is highly adopted. Equally, from a southern and eastern African perspective, Dzvimbo (1994) examined the qualitative research paradigm in education, demonstrating the dominance of and preference for "positivistic paradigm and traditional quantitative research design". 
This preference for quantitative research in the BE (Carter and Fortune 2004) is also reported in BE research in West Africa (Ejohwomu \& Oshodi 2014; Laryea and Leiringer 2012). Laryea and Leiringer (2012) found that from 2009 to 2011, the number of statistical survey studies published in the proceedings of a conference series (name purposely withheld) in West Africa, is four times more than studies that have used other strategies; specifically, 108 of the 188 papers were based on quantitative surveys, and of the 108 papers, 80 used questionnaires in data collection. They also found that experimental strategy studies accounted for 26 papers, while mathematical and analytical modelling, literature surveys and an unspecified paper accounted for 5, 24 and 11 papers respectively. In addition, Laryea and Leiringer (2012) found that only 9 studies adopted a case study strategy, while 4 studies used archival research. Laryea and Leiringer (2012) did however not specify the research approaches of the case studies and archival research strategies examined.

Therefore, given that a case study can be of a qualitative, a quantitative, or a mixed methods approach; a literature survey of qualitative or quantitative approach, archival research quantitative and qualitative approaches, and the unspecified of any research paradigm, the extent that the QA is represented in the nine case studies, the 24 literature surveys, four archival research studies and the 11 that were unspecified in Laryea and Leiringer (2012) are not known. Consequently, it is naive to conclude that the QA to research is underrepresented in the case studies, literature surveys, the unspecified studies, and archival research study.

Nonetheless, for the following reasons it is logical to deduce that the QA to research is underrepresented in Nigeria's BE research and that the quantitative approach is dominant. First, although the research approaches of the studies examined by Laryea and Leiringer (2012) are unknown, they are just approximately $26 \%$ of all the papers documented in the conference series, while the quantitative research approach papers constitute approximately $74 \%$, and the qualitative research approach studies constitute less than $1 \%$ of the papers presented. Thus, a case is made for the underrepresentation of the qualitative approach to research. Secondly, the position that the QA is underrepresented is emphasised by the premise that only one paper employing flexible research design (that is action research $=0$, grounded theory $=1$, ethnography $=0$ ), which mainly of the QA, was presented at the above-mentioned conference series (Laryea \& Leiringer 2012).

The authors consider the results of the study by Laryea and Leiringer (2012) as indicative and not absolute, because firstly, although the papers that have been published in the conference series to "...reflect the constitution and configuration of BE academic field in the region" (Laryea \& Leiringer 2012:803), some of the papers may not be based on research projects in West Africa and conclusions based on one conference series may be questionable. Secondly, there are other conferences that academics in West Africa attend.

Another study by Ejohwomu and Oshodi (2014) further confirms that qualitative strategies are underrepresented in BE research in Nigeria. Ejohwomu and Oshodi (2014) examined $\mathrm{PhD}$ research undertaken in seven departments of building and quantity surveying at Nigerian universities, from 1984 to 2012 and found that no $\mathrm{PhD}$ study adopted action research, archival research, ethnography or grounded theory. Only approximately 3\% adopted a case study strategy (again, it is not known whether 
the case study research is based on the mixed methods, quantitative, or qualitative approach), 10\% experiment, 30\% modelling and approximately 57\% statistical survey strategy. These findings suggest a lack of attention over the years to qualitative research, and the dominance of quantitative approach to research (at $\mathrm{PhD}$ level) in Nigeria's BE.

Hughes (2010) posits that it is necessary to find out whether academics are teaching and doing the right things in terms of guiding students towards the right research approach. Drawing on the premise established so far, the study sought to establish why the qualitative approach is underutilised in BE research in West Africa, the level of recognition accorded to qualitative research, and whether the disposition of academics towards qualitative research approach, impacts on the students' choice of research methodology.

\section{Implications of overlooking qualitative research strategies}

There is evidence that the implication of overlooking the QA to research is not only limited to developing countries. Authors such as Crossley and Vulliamy (1996), Dainty (2007A), Hughes (2010), and Laryea and Leiringer (2012) have established the implications of the predominance of quantitative research. The implications include research questions being wrongly tackled, some are left unaddressed and as a result, the findings of some studies may not be reliable (Crossley \& Vulliamy 1996); inadequate research methods in tackling research questions (Dzvimbo 1994; Hughes 2010; and Laryea \& Leiringer 2012); impact on research scholarship and practice, and the questionable knowledge of BE academics in industry practice (Dainty 2007A). This is despite earlier arguments by scholars such as Crossley and Vulliamy (1996), promoting the suitability of qualitative research in developing countries because of the low level of literacy and numeracy in these regions. While literacy and numeracy may have increased over the years, Crossley and Vulliamy (1996) posit that the potential of qualitative research still makes a case for it. Hughes (2010) acknowledges that there is a common assumption among novice researchers that questionnaire surveys are always needed for social science research.

\section{METHODOLOGY}

Considering the ability of qualitative research in answering 'why', 'how' and 'what' research questions (Erikkson \& Kovalainen 2008), and that this study sought to gain an in-depth understanding of a social phenomenon, the QA was adopted. As qualitative research is prone to bias, and subjective, some steps such as member checking, peer debriefing, triangulation of sources, and pretesting of the data collection instruments, were undertaken to ensure that the research findings are valid. The latter involved pretesting the data collection instrument to two academics and one student after which it was revised prior to the main data collection exercise. Prior to pretesting the data collection instrument for use in a survey, it is pertinent to ensure that the interview protocol is usable and addresses the research questions, and that it is in line with the stated aim of the research (Castillo-Montoya 2016). The interview guide was developed, based on the following: the opinions and experiences of the investigators, informal discussions with peers, literature on the representation of research paradigms both in the $\mathrm{BE}$ and outside the $\mathrm{BE}$, and the implication of overlooking qualitative research strategies. This process was initiated by the main 
investigator reading articles in the area of interest and then looking at their reference list for further reading, so as to assemble a body of literature; this is called citation pearl searching. Additionally, there were themes that emerged while writing up areas such as the discussion section of the paper that resulted in searching literature databases.

In addition to pretesting the interview protocol, some other steps taken to ensure that the interview protocol is usable and consistent with the systemic interview protocol refinement framework reported in Castillo-Montoya (2016) are as follows: "Ensuring interview questions align with research questions; construction- and inquiry-based conversation; receiving feedback on the interview protocol; piloting interview protocol." This not only helps in improving the data collection instrument, but it also helps to improve the quality of the data (Castillo-Montoya 2016). The aforesaid process was not set up in matrix form as Castillo-Montoya (2016) notes. This helped to show any gaps in addressing the research questions or where adequate interview questions addressed a particular research question. Castillo-Montoya (2016) demonstrates that contexts, such as social and political should be considered when creating interview questions from the research questions.

Also, efforts were made to ensure that the questions facilitated a conversation. This is consistent with the second phase of the said framework by Castillo-Montoya (2016). In this phase, in terms of the contexts, the questions were phased in a manner that the interviewees would understand. This involved using some local, everyday language and terms, incorporating some issues, which are commonplace in Nigeria, in the questions, and not being oblivious of the environment. The next phase involved getting feedback on the interview protocol (Castillo-Montoya 2016). While not all the steps noted in Castillo-Montoya 2016) were followed, the steps taken in this current study include: having colleagues read the interview protocol aloud and testing it to see how easy it is to answer the questions and how the questions flow, after which they would give feedback to the investigators; having the investigators individually go through the interview protocols by reading it aloud; and having the investigators discuss the interview protocol feedback and reviewing the protocol. The triangulation process involved collecting data from three $\mathrm{PhD}$ students and 12 academics from various BE departments in Nigeria. The questions that the students were asked triangulated the questions that the academics were asked. Triangulation involves using more than one data source and collaborating the evidence to support or refute each other. In terms of member checking, it involves sending the transcript of the interviews to the respondents to validate, so as to ensure the true representation of the views of the respondents (see Lietz, Langer, and Furman 2006).

The sample framework was taken from two international conference series; one held in Nigeria and one held in Ghana. Among the reasons for selecting one conference series were a lack of resources and the fact that the study is exploratory. A review of papers presented at the BE international conferences covering a two-year period (2013 and 2014), that was jointly hosted and organised by Ghana, Nigeria, and South Africa, revealed that the QA was underrepresented and that quantitative research dominant within all 93 papers in the two-conference series proceedings.

Ethical issues were considered in the current research. Introductory letters were sent by email to the respondents explaining the research project, assuring them of 
anonymity, and informing them that they could withdraw from the research (even during the interviews) up until two weeks from the day the data was collected. Respondents were also told how the data would be used. Eighty conference participants and nine $\mathrm{PhD}$ students were invited to take part in the study. The criterion for the invitation was an academic post at a Nigerian higher education institution or at a research institution. Information about the prospective respondents was verified through the various institutions to which they were affiliated. Additionally, respondent particulars were cross-checked through an Internet search. At the end of the study period, 15 responses were obtained. While it can be argued that drawing reliable conclusions based on 15 data points is questionable, it should be noted that no claim is made in this paper that data saturation was attained in the reported study. That a study is not saturated does not mean that the findings are unreliable, but rather that there is room for further exploration (O'Reilly \& Parker 2012). Exploratory studies report on small samples as small as five, for example Okorie et al. (2014). Nonetheless, in addition to being an exploratory study, which Flick (2014) views can be conducted with a sample of five (also see Okorie et al. 2014), the current study may be viewed as a scoping study and it reports on a pertinent subject that academics may find beneficial. The interest in qualitative research in general is not in the random selection of research materials, but rather, the interest is in the cases (Flick 2009).

Interviews and e-interviews were used in data collection. An e-interview is an emerging data collection method (see Bampton \& Cowton 2002) that involves the collection of data through exchange of emails between the respondents and the investigators. All the questions in the e-interviews were sent out at once and where required, further information or clarification was obtained through subsequent emails. However, the responses of one respondent who did not reply to the follow-up emails were not used. Some demographic data were collected through exchange of emails and reading up about the participants on the Internet. The questions asked, covered areas that included the understanding of the respondents about the QA in research, the QA in Nigeria's BE, and the underrepresentation of the QA. These questions were aimed at assessing the understanding of the respondents regarding the QA and their views and experiences of the QA in Nigeria's BE research. Other questions centred on the disposition of academics towards the QA and the impact of this disposition on academics and the students' choice of research methodology. These questions were aimed at understanding the disposition of academics to the QA and the implication of this disposition towards the use of the QA in research. There were also other questions aimed at explaining why the QA is underrepresented in Nigeria's BE research. All the questions were designed mainly to collect data on the experiences of the respondents.

The following questions were addressed to the academics: (I) In your experience, please comment on qualitative methods and analysis in the built environment research in Nigeria? (II) Describe the level of use or adoption of qualitative approach to research in Nigeria's BE (optional if not answered in I); (III) Based on your experience, describe what can explain the underrepresentation of qualitative approach to research in Nigeria's BE, for example, the academic background; (IV) Please describe your understanding of the suitability of qualitative research in addressing issues in BE context; (V) Describe how your previously described perceptions on the suitability of qualitative research in IV have influenced your actions in terms of the adoption of qualitative approach in research; (VI) Describe what you have done 
because of your perceptions in IV in terms of qualitative research; (VII) In your experience, how have your views (in IV) on qualitative research impacted on: what students are taught? your choice of research methodology? your research output? and your recognition of qualitative research?

The interviews were conducted at the convenience of the participants and questions were not asked in a confrontational manner. The interviewer, the lead author, ensured that there was a good rapport between him and the participants so as to make them relaxed. He assured the participants that although he is affiliated to a higher institutions in the UK, he is a Nigerian and has a good understanding of issues in the country hence they can 'open up' to him. The authors view that this may make the participants share information that they have withheld in the bid to protect the image of academics in Nigeria. During the interviews, the probing techniques included: realigning the participants when they unknowingly deviate from the answer by repeating the questions; asking the respondents to expand on points where needed; rephrasing the questions when relevant; and avoiding leading questions. The interviews (which lasted between 31-43 minutes) were recorded and transcribed verbatim. The data collected from both methods were analysed thematically, using software (NVivo for Mac). The field notes of the interviews were also factored in. Dainty, Bagilhole, and Neale (2000) acknowledge that the use of software analysis in QA reduces bias and supports methodological transparency. This will in turn improve the trustworthiness of the research. The data analysis, which involved exploring latent and manifest meaning, started at the data collection stage, because purposeful sampling was adopted. This helped in producing a thematic map of the visual presentation of the codes and categories and their relationships (Vaismoradi, Turnnen and Bondas 2013). Typically, after the first few interviews were conducted, the analysis commenced, building on the six phases of analysis, being: familiarisation with the data; initial codes; developing or searching for themes; reviewing themes; defining and naming themes; and writing up, in the thematic analysis format presented by Braun and Clarke (2006).

Familiarisation with the data involved reading the transcripts, the e-interviews scripts and field notes many times, immersing the main investigator in the data. At this stage, the main investigator started making notes and the direction of storyline of the data started to form. The next phase, initial codes, involved asking questions such as: what is happening here?; What is the respondent saying?; What information is missing here? (Basit 2010). Other steps here include: looking for commonly used words suggesting conceptual categories (Bowen 2008); reading the sentences one after the other and coding line-by-line to extract the latent and manifest content; alongside constantly comparing coded text with other coded text. The latter ensures consistency in the coding process. This stage involved a combination of inductive and deductive approaches. The deductive approach was informed by the literature review. This is consistent with data-driven and theory-driven coding where the latter is an inductive approach and the former a deductive approach (Braun \& Clarke 2006). During the coding process there were codes that were interrelated both as per latent and manifest meaning, so they were coded, annotated or reported as memoranda. After the initial codes were allocated, the codes were re-examined, searching for further codes, eliminating and combining the codes where necessary, and developing themes and subthemes. The level of consistency of the codes informed the combined themes and subthemes. 
Visual presentation in NVivo for Mac helped in sorting the codes. At this stage the relationships between codes and their categories and subcategories were explored. There were codes that did not fit into any subcategory or category; thus, they were classed in a category called outliers. At the end of this phase, the main themes referred to as candidate themes by Braun and Clarke (2006) - were now clearer, but they needed to be refined and addressed in the succeeding phase, 'reviewing themes'. This resulted in some themes being eliminated and some merging to form existing themes. Explanations for eliminations and mergers include insufficient codes or data, inconsistence in the data, and strong latent or manifest meaning with another item. This process involved reading each theme against all the collated data to see if there was consistency between them, after which the second level of checking each theme against the entire data set for consistency or further coding was undertaken - see Braun and Clarke (2006). At this stage, further coding and refinement of the themes occurred, until it was not sufficiently significant to change the meaning or message of the themes. Defining and naming the themes was the fifth stage which involved organising all the collated data in each theme and ensuring that each theme was internally consistent and coherent, identifying interesting content, and telling a story about the theme (Braun \& Clarke 2006). The aforesaid was not treated in insolation to the overall story emanating from the data and the research questions. This defining and naming the themes also involved ensuring that the names capture the message that the theme intends to convey. The last phase involved writing up the final themes, including quotations to enhance the message received by the reader.

\section{RESULTS}

\section{Overview of the profiles of the respondents}

The respondents were academics from various $\mathrm{BE}$ disciplines in both private and public higher institutions in Nigeria. All 12 academics have a degree or equivalent and a master's degree in one or a combination of two of the following: civil engineering, building construction, architecture, quantity surveying, urban and regional planning, real estate management, and surveying and geoinformatics, housing and development, construction management, and systems engineering. Seven of the 12 respondents also have doctoral degrees in five of the above disciplines, plus construction engineering and management. Three of those who have doctorate degrees are currently doing postdoctoral research but two already have postdoctoral training. Of the remaining five without a $\mathrm{PhD}$, four are currently studying towards a $\mathrm{PhD}$ degree. Also, all first degrees or equivalent are from a Nigerian higher institution; three of the participants have a mixture of both a local Nigerian and a foreign academic background at postgraduate level, or only the latter; the others have a postgraduate degree from a developing country. It may be argued that using academics without a doctorate may skew the results, as a doctoral training is for research. It should be noted that while four of the five participants with only a first and master's degrees are studying for a $\mathrm{PhD}$, all are active in research, because conducting research counts towards Continuous Professional Development (CPD) and improves the chances for academic promotion in the Nigerian education sector. These academics have experience in academia ranging from four to over 15 years. It is important to note that the teaching activities of some these academics cut across various departments within the BE. The students were two MPhil students (from building construction and project management disciplines) and one first-degree (in 
quantity surveying) graduate from local Nigerian higher education institutions, but all are studying towards a $\mathrm{PhD}$. Again, while it may be argued that using students without a doctorate may skew the results, the inclusion of the above students is to get their perspectives as students, not as academics. It should also be noted that they are research students, some having already obtained research degrees. All six geopolitical zones in Nigeria are represented by the respondents in this study through either their current or their previous place of work (involving academic activities).

\section{Participants knowledge of qualitative research}

Responses provided to questions that assessed the respondents understanding of qualitative research showed that the academics have a very good understanding of the methodology. Also, the same can be said for all but one of the students.

\section{The use of qualitative research in Nigeria's BE}

When asked to describe the level of use and adoption of qualitative research in Nigeria's BE, the responses of all the respondents (whether from social science or engineering discipline) evidence a consensus that the QA is underrepresented in Nigeria's BE. In addition, the QA is viewed as being inadequate for BE research and assumed to be inappropriate. Typically, some respondents stated that: "Qualitative research is at infancy"; "... qualitative techniques are almost non-existent"; "Quantitative research remains popular in developing countries"; "...academics are generally not well grounded in qualitative research procedure". Some respondents noted that limited QA studies are skewed to one qualitative research approach or method.

\section{The disposition of academics towards a qualitative research approach}

While there is limited evidence of a constructive disposition toward the qualitative research approach, all the respondents alluded to the negative disposition of academics towards qualitative research (see Table 1). These negative dispositions align towards 'what' questions which in turn result in quantitative methods. In addition to Table 1 there are some significant experiences of the respondents: for example, one academic opined that the strong bias towards the quantitative research approach means that students are awarded low marks for adopting a QA.

Table 1: Summary of results on the disposition of academics towards qualitative research approach

\begin{tabular}{ll}
\hline Themes & Evidence from interviews and e-interviews \\
\hline - Credibility & QA is viewed as not scientific enough and not accurate for the built environment. \\
- Bias against a & Preference for quantitative topics. \\
QA & Aligning students' research topics to quantitative research. \\
& Insisting on incorporating quantitative methods in qualitative studies. \\
& Discouraging students from adopting qualitative research. \\
- Convenience & Research is conducted if convenient. For example, qualitative research \\
& is viewed as cumbersome, time consuming and hard to analyse, and not \\
- Amenable & encouraged by the contexts of Nigeria, so it is not worth conducting \\
& There is evidence alluding and/or demonstrating that the academia appears to be \\
- Commitment to & research. \\
Providing hands-on QA training to students. \\
a QA
\end{tabular}




\begin{tabular}{ll}
\hline - Pursuit for & While there is evidence of a small number of academics committing to QA, there \\
currency in & is plenty evidence of no zeal for change in achieving currency in a worldwide \\
research & research trend. This is because the following obsolete norms are still accepted: that \\
& quantitative methods must be part of a viva to be successful; some journals, \\
conferences and many in the industry do not accept qualitative research.
\end{tabular}

Two academics noted that external influences can explain the bias against a QA and suggested the theme 'Pursuit for currency in research' in Table 1. According to the respondents, their students were told by internal examiners to triangulate their QA output with questionnaires, so as to ensure that they do not encounter problems with the external examiners; this, despite the high standard of the qualitative work. An explanation for this may be deduced from the theme 'credibility' in Table 1. In addition to the points in Table 1 on 'credibility', some respondents explained that the quality of some QA papers are questioned because the methodologies are not clearly explained.

In addition, while all respondents note or suggest that they are discouraged from adopting a QA, there is evidence of the coercion to use a mixed-methods research approach in lieu of a QA. For instance, one academic (a quantity surveyor) stated: "In my experience, I find that academics try to discourage students from qualitative research but where a student expresses a good understanding of the qualitative approach and can demonstrate competence, the academics would often 'compromise' by insisting that the student adopts a mixed-method approach (triangulation)".

On the other end of the spectrum, the few academics that were committed and have a strong passion for the QA (see Table 1), showed a consistent pattern of independently promoting the paradigm without bias. They also noted a strong level of acceptance from the students. One academic (of civil engineering and construction engineering and management backgrounds) stated: "Sixty percent of my research students use qualitative research methods. When my project topics are advertised I let the students brainstorm on the most suitable method to adopt." This same respondent also provides hands-on training to students in addition to training the students in qualitative methods, such as interviews - he lends his recording device to the students, and supervises two interview sessions.

Using matrix-coding query, a group analysis between academics with a fully Nigerian education background, and those with a mixture of Nigerian and developed economy education background, was done. This stems from the points raised by some respondents on the influence of a developed-economy educational background and the fact that the QA has emerged from, and is employed more often in BE research in developed countries. The enquiry found strong evidence of a commitment to a full QA among a few academics with a mixture of Nigeria and developed country educational background. The academics with a mixture of Nigeria and developed country educational background showed that many academics (excluding themselves) are biased against the QA: many academics do not see QA as credible and would thus maintain the status quo, discouraging students from adopting QA. In the same vein, those with a full Nigerian educational background identified all the above and went further to identify issues relating to 'convenience', 'agreeable', and 'Pursuit for currency in research'. However, one of these respondents demonstrated a strong personal commitment to a full QA to research. 


\section{Implications of the disposition of academics towards a qualitative research approach}

The implication of a negative disposition (see Table 1) means that some students have a wrong understanding of qualitative research. However, one student stated that for him this has been corrected through independent reading online. According to the student, the negative disposition of her lecturers towards qualitative research, made her to explore the QA independently. This suggests that this student possesses some elements of academic leadership. Table 2 shows themes related to both a positive and a negative disposition towards a QA.

From the 'skewness to quantitative approach' theme (see Table 2), it can be inferred that the quantitative approach is largely seen as acceptable and its generally encouraged implies that knowledge and research output is skewed towards the quantitative paradigm. In addition, Table 2 reveals that a significant number of students and academics embrace the quantitative research approach with implications not limited to questionable quantitative research output as one respondent stated: "Many research on BE are behavioural-oriented, yet people use quantitative methods instead of qualitative methods. This always produced misleading results and faulty recommendations."

Table 2: Summary of the implications of the disposition of academics towards QA

\begin{tabular}{ll}
\hline Themes & Evidence from interviews and e-interviews \\
\hline - Increased workload & Modifying the research topics of students to a quantitative approach. \\
& Insisting that students include quantitative methods after adopting a \\
& QA. \\
& Canceling the research topics of students. \\
& Quantitative research is largely acceptable and mainly encouraged. \\
- Skewness to & Producing academic followers and not academic leaders. \\
- Luantitative paradigm & Clinging to the obsolete understanding that only a quantitative \\
& paradigm is sound. \\
& Oblivious and/or resistance to emerging/cutting-edge ideas or \\
& methods. \\
- Championing paradigm & Hands-on training and supervision of students using a QA. \\
shift & Providing interview recording device to students. \\
- Questionable knowledge & The dominance of quantitative research implies that some issues will \\
output & be wrongly tackled, producing questionable knowledge. \\
practice & Scoring students that adopt QA low in exams \\
\hline
\end{tabular}

A significant number of the respondents opine that the BE is not in touch with current trends in the industry including academia, suggesting the need for academics to strive to learn more towards becoming research experts. For example, a respondent stated that: "It is indeed very worrying to think that over 90 percent of our research activities are embedded in quantitative research in an era where the BE challenges is such that there is increasing need to know more and more of less and less (Nano scale)".

\section{Possible explanations for the limited use of qualitative research}


The interviews and e-interviews revealed some explanations for the limited use of a QA in BE research in Nigeria. The explanations are presented in Table 3. Although the challenges in research, such as logistics, are general, they are not excusable; the responses of both students and academics demonstrate the strong negative impact of these challenges on qualitative research in Nigeria. Furthermore, the respondents noted contextual issues such as gender bias, poverty, a high level of illiteracy in society, and the disposition of the population towards research (see Table 3). However, one respondent strongly contends the above, suggesting the need for a change in the direction of teaching; he stated: "The African way of life cannot be an excuse in my opinion but I'd rather say we lack progressive academics that have refused to embrace change. We currently are churning out academic followers rather than academic leaders." Additionally, a few other respondents noted or suggested that the contexts of Nigeria are advantageous to qualitative research.

With regard to the transition from quantitative to qualitative research (Table 3), while many of the respondents blame the underrepresentation of QA on preceding academics in that instead of driving change, they resisted it; even so, a few academics noted that there have been cases where 'old hands' in the academia succumbed to a QA. As a result, according to one respondent, many academics that have been trained in quantitative research would rather stick to what they know, viewing qualitative research as unacceptable and discouraging students from using it. This attitude is then transferred to the next generation of academics, as was noted by one of the respondents. These attitudes may be explained by the responses to the themes 'norms and culture' and 'educational background' in Table 3

Table 3: Summary- possible explanations to the status quo of a QA in Nigeria's built environment research

\begin{tabular}{|c|c|}
\hline Themes & Evidence from interviews and e-interviews \\
\hline $\begin{array}{l}\text { - Challenges, } \\
\text { constraints \& } \\
\text { limitations }\end{array}$ & $\begin{array}{l}\text { Negative attitude of journals towards qualitative research. } \\
\text { Socio-cultural, economic, political \& institutional contexts. } \\
\text { Limitations of qualitative research, e.g. credibility of data. } \\
\text { General constraints and challenges in research. }\end{array}$ \\
\hline $\begin{array}{l}\text { - Educational } \\
\text { background }\end{array}$ & $\begin{array}{l}\text { Many respondents view the science or engineering background of academics } \\
\text { as highly contributory to their strong attachment to quantitative research. } \\
\text { While many also demonstrated the influence of senior academics, few noted } \\
\text { the influence locality of education and direction of teaching. }\end{array}$ \\
\hline $\begin{array}{l}\text { - Norms and } \\
\text { culture }\end{array}$ & $\begin{array}{l}\text { The attachment to quantitative approach has been passed on from preceding } \\
\text { academics to younger ones and has become accepted. }\end{array}$ \\
\hline - Ignorance & There is lack of understanding of the potential and attributes of a QA. \\
\hline - Transition & Resistance to change; maintaining the status quo. \\
\hline
\end{tabular}

The results show that a significant number of respondents through their suggestions, demonstrations and direct quotations corroborate the premise of 'educational background' as explaining the QA in Nigeria. Further explanation is given by the theme 'locality of education' in Table 3; few respondents (from social science and/or engineering disciplines) opined that academics trained abroad are more likely to embrace the QA to research. Indeed, one academic stated that the application of the QA is low because: “... a large portion of the BE academics in Nigeria are locally trained. Hence, there was no avenue for cross-fertilization of ideas about research methods. "However, while this argument may hold in general, as there is evidence in this study of students being discouraged from adopting a QA (see Table 1), one of the respondents who is committed to qualitative research, is locally trained. 
Furthermore, the fact that the $\mathrm{BE}$ is made up of more of science and engineering disciplines as opposed to social science and art, also explains the theme 'educational background', as noted by many of the respondents from social science and/or engineering disciplines. A respondent stated that: "The BE is considered to be more of a science based field rather than arts or social science and so quantitative research has been the more commonly accepted approach. Consequently, I would describe qualitative research as emerging - it is wrongly perceived to be inappropriate, unpopular and much too subjective for a 'scientific' mind."

Using matrix-coding query, a group analysis between academics with a fully Nigerian educational background, and those with a mixture of local Nigerian and foreign academic background, was conducted. This query helped in distilling what each of these two groups had to say about the themes in Table 3. In terms of 'educational background' those with a fully local Nigerian educational background spoke a lot about science/engineering background and a little about the influence of the locality of the educational background of academics. Those with a mixture of Nigerian and foreign educational background spoke a little about the locality of the educational background of academics, and also spoke a little about the science/engineering background of academics. Both groups also highlighted the influence that senior academics have on the direction of research and teaching. In terms of 'ignorance' (Table 3), quite a lot of academics with a fully Nigerian academic background spoke about a lack of understanding of the QA in various ways. Those academics with a mixture of Nigerian and foreign academic backgrounds also, but to a lesser extent expressed the above-mentioned comment, but seem to understand why their colleagues are ignorant of the QA, attributing it to 'norms and culture' (see Table 3).

\section{Relationships among themes}

There are various types of relationships observed among the themes. For instance, 'convenience' in Table 1 has a one way and/or symmetrical relationship with 'challenges, constraints and limitations' in Table 3, in that the latter explains the former and/or contributes to the former. In the same vein, 'background of education' in Table 3 has a one way and/or symmetrical relationship with 'bias towards qualitative approach' in Table 1, in that the latter explains the former and/or contributes to the former.

\section{DISCUSSION}

The profile of the respondents and the assessment of their understanding of QA demonstrate their credibility, alluding to the reliability of the information provided by the respondents.

\section{The status quo of the qualitative research approach and disposition of academics towards it}

The findings of this study are aligned to the position of authors in that the QA is underrepresented in the BE; this is also the case in developing countries and outside the BE field (Carter \& Fortune 2004; Dainty, Bagilhole, and Neale 1997; Danity 2007A, B; Dzvimbo 1994; Ejohwomu \& Oshodi 2014; Hoepfl 1997; Laryea \& Leiringer 2012; Loosemore, Hall, and Dainty 1996; Panas \& Pantouvakis 2010; Zuga 
1994). The findings also provide evidence to support the assertion that the QA is emerging at a slow pace. This is because, despite the consensus on the under-adoption of the QA and the unfavourable disposition towards it, there is evidence that the QA is being recognized as a full-fledged research methodology. Their disposition towards QA means that academics in Nigeria's BE can be categorised into five groups: those passionate about a QA and are fully immersed in it; those that insist on a quantitative approach; those that are indifferent, but will initially encourage a quantitative approach; those that are indifferent to a QA, and feel that research must have quantitative elements to produce valid research; and those that see the QA as an inconvenience, not credible and unacceptable, and not worth conducting. The negative disposition of the $\mathrm{BE}$ academics towards QA suggest that the academics may align toward 'what' research questions thus towards quantitative approach to research. This does not mean that there cannot be 'what' questions to be addressed with qualitative methods, for example see (Erikkson and Kovalainen 2008). Based on the points outlined above, the adoption of quantitative methods is then more like a culture where the forefathers and senior academics are responsible for a quantitative culture. This is consistent with the position of Carter and Fortune (2004) noted elsewhere in this paper. However, it is possible to argue that describing the negative disposition of some academics to a QA as cultural, is inappropriate because not all academics accept the norm.

\section{Implications of the disposition of academia towards a qualitative research approach}

There are some significant points to be noted about some of the themes and findings under this topic (see Table 2). For instance, 'increased workload' and 'marking students low' 'discouraged from adopting QA' have indirect implications, such as psychological effects. This is in addition to the implication of untapped potential in students and academics which is smothered when they are discouraged from adopting a QA. However, to be fair to the academics, there are possible reasons for unenthusiastic attitudes to the QA. Typically, insisting that students add quantitative to the output of their QA work (which will increase the workload) may occur because the academics belongs to one of the groups in the preceding sub-section, therefore, research must have elements of quantitative to make it a valid research. Canceling the topic may happen because the academic finds QA too challenging and cumbersome, and perhaps considers it unachievable within the limited time available. Also, the academic may be unfamiliar with the implementation of the qualitative research approach. Scoring students that adopt QA with low marks could arise because the students may not have done the research very well, especially considering that a QA requires more skills and knowledge than the quantitative approach (Bubaker et al. 2005).

Relating to 'questionable knowledge' (see Table 2), this is consistent with the arguments of Crossley and Vulliamy (1996), Dzvimbo (1994) and Hughes (2010). Adhering to a particular research paradigm means that some research questions will be left unaddressed, or will be wrongly tackled and the problems that could have been solved by the research will continue to proliferate. This is not limited to the study population, but also applicable to the holistic environment as found in literature, (see Carter \& Fortune, 2004; and Crossley \& Vulliamy, 1996). However, the favourable attitudes of a few academics towards a QA bodes well; where one academic narrates working 'hand-in-hand' with students, equipping them with the necessary tools, can 
be viewed as an individual's effort towards ensuring currency in education. According to the findings of Amaratunga and Senarantne (2009), the individual commitment of academics in research-biased departments fosters "research to teaching transfer." Additionally, the favourable attitudes of few academics towards a QA show that they are not oblivious of their environment and have developed currency in research.

\section{Possible explanations for the status quo of qualitative research}

Considering the theme 'challenges, limitations and constraints' (Table 3), Editors have been known to turn down qualitative research approach papers and are unwilling to commit to qualitative research (Dzvimbo 1994), but this has not discouraged academic leaders (Putten 2006). Thus, if there is a good understanding of the potential of the QA in Nigeria, it should challenge academics. Equally important are contextual issues, such as the fact that gender bias is consistent with the findings of Kaewsri and Tongthong (2014), who report gender bias towards female in the construction industry due to intrinsic and extrinsic factors, resulting in instances where 11 out of 12 respondents have experienced slow career progression. An example of an intrinsic factor is where a female voluntarily decides not to work on construction sites because of their hazardous nature, while a typical extrinsic factor would be an instance where females want to work on construction sites, but are allocated to positions in offices. Conversely, there are instances where gender bias is in favour of the female (Maina 2015). It is subject to research to provide evidence whether gender bias is obvious in the research environment. Furthermore, Dzvimbo (1994) also noted the influence of the political environment of some African countries on qualitative research, which is consistent with political influence mentioned in Table 3 . However, while contextual issues can be challenging, they should not be a limitation. In support, Loosemore and Chin Chin (2000) encourage researchers to exploit the occupational cultural diversity of their respondents.

It is observed that engineering and the science backgrounds in Nigeria's BE play a significant role in the under-adoption of a QA in Nigeria's BE research. This is consistent with the position of Loosemore and Chin Chin (2000) who present a treatise on the impact of occupational bias in construction research. Occupational bias has to some extent established a norm and culture of quantitative approach to research. It is imperative to ask if it is likely that academics with a pure social science background would be more attracted to a QA because of their academic background. This is an area that further studies can explore. The authors of this paper view that more attention to QA to research is a substantially stronger recommendation than the academic training and background of that academic.

\section{CONCLUSION}

This study advances the understanding of qualitative research at large, using Nigeria's $\mathrm{BE}$ as a case study. In providing supporting qualitative evidence to the existing quantitative indication of the underrepresentation of a QA in research, there is also substantiation of the value of a QA to Nigeria's BE research in this study. Among the findings of this study are that: many academics are not amenable to currency in research; a QA is viewed as unscientific and unacceptable; a QA is only to be adopted when convenient; there is bias against qualitative research. These findings may be explained by: the education background of academics; ignorance; challenges and 
limitation due to the contexts of Nigeria inter alia; and quantitative research becoming a culture. However, there is compelling evidence showing that a small number of academics are committed to a QA. The disposition of the academics can be explained (as seen in the discussion) by a framework consisting of five categories thus: those passionate about a QA and are fully immersed in it; those that insist it must be a quantitative study; those that are indifferent, but will initially encourage a quantitative approach; those that are indifferent to a QA, and view that research must have quantitative elements to produce valid research; those that see the QA as an inconvenience, not credible and unacceptable, and not worth conducting. Scoping this study back to the international audience, it can be argued that if the result of this study is reflective of the BE in general, then a QA is still underrepresented, but gradually improving. The aforesaid outline may also contribute to explaining the disposition of academics towards a QA, or for conducting further research in this area.

As a result of the disposition of the academics, a QA is not always encouraged in Nigeria's BE, where research output and knowledge is skewed towards the quantitative paradigm, unwittingly reducing industry contribution to currency and perhaps causing the industry to be oblivious to its environment. Furthermore, while the research output and knowledge in the academia are questionable, there is an increased workload for students and strong steps, such as awarding low marks for students who follow a QA, further discouraging the adoption of a QA. This in turn impacts on achieving a balanced methodological outlook. Scoping these implications of the disposition of academia towards a QA to the international audience, it is subject to research to show if the reported implications above are replicated outside the study population. However, the findings here indicate the possibility of academics discouraging students from adopting a QA to research. This study observed that there is need for a paradigm shift by professional institutions and senior academics to a QA in research, so as to create a balanced methodological outlook in Nigeria's BE research. While Nigeria is used as a case study in this research, BE research in general may find this study useful as it can be employed as a step towards achieving a balanced methodological outlook.

In support of development and achieving currency, stakeholders in academia in developing countries need to set up strategies that address the issues established in this study. The authors of the current paper hope that a study of this nature will form the basis for a shift in the disposition of academics in Nigeria's BE research towards a QA, but bearing in mind that the methods to be adopted should be fit-for-purpose, for instance, if research questions start with 'how' and/or 'why', seeking to deeply understand the social phenomena, a QA is more appropriate. In other words, adopting an adequate research approach which is determined by the research questions and not adhering to any particular research approach would ensure a balanced methodological outlook in not only the BE in developing countries, but also in developed countries and other disciplines. With respect to the matrix-coding query reported in this study, it is up to the reader to interpret and apply the result within their context. However, the authors of this paper suggest that the results of matrix-coding query should be viewed as indicative and not absolute.

This study faced some limitations. Firstly, the investigators did not observe the object of study through ethnography, and such a method might have provided additional knowledge. Further study that observes the object of study through ethnography is 
recommended. Secondly, as the sample is from two international conference series the results will be skewed towards researchers who attend those conferences and caution must be taken when generalising the results to other the population of BE academics in Nigeria. Lastly, the small sample of the study makes drawing strong conclusions from the study challenging, however, the study should be treated as a scoping study with further research of larger sample recommended. Also, further studies can also seek to address the aforesaid and to understand why few academics in Nigeria's BE have a strong commitment to a QA to research in an environment where it is under adopted.

Emphatically, no claim is made in this paper of the underrepresentation of QA in BE research in all countries. Rather, there is evidence that quantitative approach to research is dominant, with overreliance on the quantitative survey, and that a QA to research is underrepresented in Nigeria's BE research. The authors of this paper consciously simplified the research by comparing quantitative and qualitative research and not adopting epistemological terms such as positivist versus non-positivist. Another reason for simplifying the research is for the benefit of novice researchers or novice students. Comparing quantitative and qualitative research means that only these paradigms were covered, thus pragmatic researchers were not covered; it can be argued that examining pragmatism is outside the scope of this study (and not the research question) as pragmatic researchers are not biased towards any paradigm.

\section{REFERENCES}

- Amaratunga, D. \& Senarantne, S. (2009) Principles of integration research into teaching in higher education: built environment perspective. International Journal of Construction Education and Research, 5(3), 220-232.

- Bampton, R. \& Cowton, C.J. (2002). The e-Interview forum qualitative, Socialforschung/Forum: Qualitative Social Research, 3 (2) Art 9. Retrieved on 03/01/16: http://www.qualitative-research.net/index.php/fqs/article/view/848/1843

- Basit, T. (2003) Manual or Electric? The role of coding in qualitative data analysis, Educational Research, 45(2), 143-154.

- Braun, V. \& Clarke, V. (2006) Using thematic analysis in psychology, Qualitative Research in Psychology, 3:2, 77-101

- Bubaker, S., Balakrishnan, P. \& Bernadine, C. (2005) Qualitative case study research in Africa and Asia: Challenges and prospects. Procs. 3rd International Qualitative Research Convention, Malaysia, 1-13,

- Carter, K. \& Fortune, C. (2004) Issues with data collection methods in construction management research. In: Khosrowshahi, F (Ed.), Proceedings 20th Annual ARCOM Conference, 1-3 September 2004, Edinburgh, UK, (2), 939-46.

- Castillo-Montoya, M. (2016) Preparing for Interview Research: The Interview Protocol Refinement Framework. The Qualitative Report, 21(5) 811-831

- Creswell, J. W. (2014) Research design: Qualitative, quantitative, and mixed methods approaches, $\left(4^{\text {th }}\right.$ ed.), London, Sage publications.

- Crossley, M. \& Vulliamy, G. (1996) Issues and trends in qualitative research: Potential for developing countries. International Journal of Educational Development, 16(4): 439-448.

- Dainty, A.R.J. (2007A) A call for methodological pluralism in built environment research. In Egbu, C.O. and Tong, M.K.L. (Eds), Proceedings of 3rd Scottish 
Conference for Postgraduate Researchers in the Built \& Natural Environment (PRoBE), Glasgow Caledonian University, Glasgow, 20-22 November, pp. 1-10.

- Dainty, A.R.J. (2007B) A review and critique of construction management research methods, in Hughes, W. (ed.) Proceedings of Construction Management and Economics 25th Anniversary Conference, University of Reading, 16-18 July, p. $1533-1543$

- Dainty, A. R. J., Bagilhole, B. M., \& Neale, R. H. (1997) Analytical strategies for dealing with qualitative data in construction management research. In Stephenson, P. (Ed) Proceedings 13th Annual ARCOM Conference, 15-17 September 1997, King's College, Cambridge, (2), 484-493.

- Dainty, A. R. J., Bagilhole, B. M., \& Neale, R. H. (2000) Computer aided analysis of qualitative data in construction management research. Building Research and Information 28(4), 226-233. DOI: 10.1080/09613210050073689

- Dzvimbo, K. (1994) Qualitative research in African education: Notes and comments from Southern and Eastern Africa. Qualitative studies in Education, 7(3), 197-205.

- Ejohwomu, O.A. \& Oshodi, O.S. (2014) A review of construction management and economics research outputs in Nigeria: To towards a sustainable future. Journal of Construction Project Management and Innovation, 4, (S1): 900-905.

- Erikkson, P. \& Kovalainen, A. (2008) Qualitative research in business research. Thousand Oak. CA. Sage publications.

- Flick, U. (2009) Managing quality in qualitative research, London: Sage Publication.

- Flick, U. (2014) An introduction to qualitative research. (5th ed.). London: Sage publication.

- Hesse-Biber, S. N. \& Leavy, P. L. (2011) The practice of qualitative research, $2^{\text {nd }}$ edition, London: Sage Publication.

- Hoepfl, M. C. (1997) Choosing qualitative research: A primer for technology education researchers. Journal of Technology Education, 9(1), 47-63.

- Hughes, W. (2010) Built Environment education, research and practice: Integrating diverse interests to make an impact. In: Laryea, S., Leiringer, R. and Hughes, W. (Eds) Procs West Africa Built Environment Research (WABER) Conference, 27-28 July, Accra, Ghana, 1-8.

- Hyari, H.K., El-Mashaleh, M.S., \& Rababeh, S.M. (2015) Framework For Managing The Traffic Impacts of Building Construction Project. Journal of Construction in Developing Countries, 20 (2) 97-113.

- Kheni, N.A. (2008). Impact of health and safety management on safety performance of small and medium-sized construction business in Ghana (Doctoral Thesis Loughborough University). Retrieved on 12/12/15: https://dspace.lboro.ac.uk/dspacejspui/bitstream/2134/4079/3/Kheni\%27

- Laryea, S. \& Leiringer, R. (2012) Built Environment research in West Africa: current trend and future direction. In: Laryea, S., Agyepong, S. A., Leiringer, R. and Hughes, W. (Eds) Procs $4^{\text {th }}$ West Africa Built Environment Research (WABER) Conference, 24-26 July, Abuja, Nigeria, 797-804.

- Lietz, C.A., Langer, C.A., \& Furman, R. (2006) Establishing trustworthiness in qualitative research in social work: Implications from a study regarding spirituality. Qualitative social works, (5): 441-458. 
- Loosemore, M., Hall, K \& Dainty, A. (1996) Innovation and courage in construction management research, Procs., $12^{\text {th }}$ Annual ARCOM Conference, 418427.

- Loosemore, M. \& Chin Chin, T. (2000) Occupational bias in construction management research. Construction Management and Economics, 18(7), 757-766.

- Kaewsri, N. \& Tongthong, T. (2014) Favorable female attributes in relation to caeer challenges of women engineers in the Thai construction industry. International Journal of Construction Education and Research, 10(3), 222-236.

- Maina, J. J. (2015) Architects and interdisciplinary research: Reflections from ethnographic and measured fieldwork: In Laryea, S. and Leiringer, R. (Eds) Procs $6^{\text {th }}$ West Africa Built Environment Research (WABER) Conference, 10-12 August, Accra, 131-145

- Okorie, V., Emuze, F., Smallwood, J. \& Van Wyk, J. (2014). A qualitative review of the health and safety leadership roles of managers in South African construction. Journal of Construction Project Management and Innovation, 4 (2), 950-965.

- O'Reilly, M. \& Parker, N. (2012) Unsatisfactory Saturation': a critical exploration of the notion of saturated sample sizes in qualitative research. Qualitative research, 12(2), 190-197.

- Panas, A. \& Pantouvakis, J. P. (2010) Evaluating research methodology in construction productivity studies. The Built and Human Environment Review, 3(SP.1) 63-85

- Putten, J.V. (2006) In Peer Review, It's Time to Stop Thinking Statistically About Qualitative Research. Teachers College Record, Retrieved from: http://papers.ssrn.com/sol3/papers.cfm?abstract_id=1699349 on 03-01-16

- Saunders, M., Lewis, P. \& Thornhill, A., (2009) Research Methods for Business Students (5th ed.), London: Prentice Hall.

- Strauss, A. \& Corbin, J. (1990) Basics of qualitative research: Grounded theory procedures and techniques. Newbury Park, CA: Sage Publication

- Umeokafor, N. (2015). A discussion of obser-view as a method of generating data in the construction industry. Civil Engineering Dimension, 17(1), 54-58.

- Vaismoradi, M., Turnnen, H. \& Bondas, T. (2013) Content analysis and thematic analysis: implications for conducting a qualitative descriptive study. Nursing and Health Science, 15, 398-405. 